\title{
Decreased sEng plasma levels in hypertensive patients with endothelial dysfunction under chronic treatment with Perindopril
}

This article was published in the following Dove Press journal:

Drug Design, Development and Therapy

\author{
Valentina Buda ${ }^{1}, *$ \\ Minodora Andor ${ }^{2, *}$ \\ Dana Emilia Baibata ${ }^{3,4}, *$ \\ Ramona Cozlac ${ }^{3,4}$ \\ Gabriela Radu ${ }^{2}$ \\ Dorina Coricovac ${ }^{5}$ \\ Corina Danciu ${ }^{6}$ \\ lonut Ledeti ${ }^{7}$ \\ Adelina Cheveresan ${ }^{8}$ \\ Cristian Nica ${ }^{9}$ \\ Paul Tuduce ${ }^{10}$ \\ Mirela Cleopatra Tomescu ${ }^{2}$ \\ 'Department of Pharmacology and Clinical \\ Pharmacy, Faculty of Pharmacy, "Victor Babeș" \\ University of Medicine and Pharmacy, Timisoara, \\ Romania; ${ }^{2}$ Department of Medical Semiotics, \\ Faculty of Medicine, "Victor Babeș" University of \\ Medicine and Pharmacy, Timisoarn, Romania; \\ ${ }^{3}$ Department of Cardiology VI, Faculty of Medicine, \\ "Vepartment of Cardiology VI, Faculty of M \\ "Victor Babeș" University of Medicine and
Pharmacy, Timisoara, Romania; ${ }^{4}$ Cardiovascular \\ Diseases Institute, Timisoara, Romania; \\ ${ }^{5}$ Department of Toxicology, Faculty of Pharmacy, \\ "Victor Babeș" University of Medicine and \\ Pharmacy, Timisoara, Romania; ${ }^{6}$ Department of \\ Pharmacyosy, Faculty of Pharmacy "Victor \\ Pharmacognosy, Faculty of Pharmacy, "Victor \\ Babeș" University of Medicine and Pharmacy, \\ Timisoara, Romania; 'Department of Physical \\ Chemistry, Faculty of Pharmacy, "Victor Babeș" \\ University of Medicine and Pharmacy, Timisoara, \\ Romania; ${ }^{8}$ Department of Pharmacology, Faculty of \\ Medicine, "Victor Babeș" University of Medicine \\ and Pharmacy, Timisoara, Romania; 'Department \\ of Surgery, Faculty of Medicine "Victor Babes" \\ Univery, \\ University of Medicine and Pharmacy, Timisoara \\ Romania; ${ }^{10}$ Department of Balneophysiotherapy, \\ Rheumatology and Rehabilitation, Faculty of \\ Medicine, Vasile Goldis Western University, Arad, \\ Romania
}

*These authors contributed equally to this work

Correspondence: Minodora Andor "Victor Babeș" University of Medicine and Pharmacy, 2nd Eftimie Murgu Street,

Timisoara, Romania

Tel +4025 6494804

$\mathrm{Fax}+40256494604$

Email andorminodora@gmail.com
Purpose: Endoglin is a transmembrane glycoprotein which plays an important role in maintaining cardiovascular homeostasis. One of its forms, soluble endoglin (sEng), a molecule with antiangiogenic properties, has been found overexpressed in patients suffering from hypercholesterolemia, diabetes mellitus and hypertension, and is proposed as a marker of endothelial damage. Accordingly, we aimed to quantify the efficacy of various antihypertensive regimens on sEng levels, in hypertensive patients with endothelial dysfunction.

Patients and methods: 323 patients were enrolled, and there were 99 patients with normal blood pressure values, 106 hypertensive patients under chronic treatment with different types of antihypertensive molecules (beta blockers, calcium channel blockers, and diuretics) in monotherapy, and 118 hypertensive patients under chronic treatment with perindopril. sEng plasma levels were quantified and were correlated with classical methods of assessing the endothelial damage.

Results: Patients under chronic treatment with perindopril had lower sEng plasma levels compared with the other group of hypertensive patients under different regimens of antihypertensive treatment (sEng: $4.73 \pm 1.39$ versus $5.63 \pm 2.33, p<0.01$ ).

Conclusion: Decreased sEng plasma levels were found in patients under chronic treatment with perindopril, when compared with other antihypertensive regimens of treatment (beta blockers, calcium channel blockers, and/or diuretics).

Keywords: ACE inhibitors, essential arterial hypertension, endothelial dysfunction, anti angiogenic therapy, endoglin

\section{Introduction}

Endothelial dysfunction (ED), a characteristic of patients with essential arterial hypertension (either as a cause or as a consequence of hypertension), plays an important role in the development of atherosclerosis and plaque formation. ${ }^{1,2}$ It is characterized among others by increased vascular permeability, which facilitates the immune cells to enter in the sub-endothelial space, producing an inflammatory process which will later contribute to the accumulation of cholesterol molecules, the early stage of atherogenesis. ${ }^{2,3}$

It was shown that soluble endoglin (sEng) plasma concentrations are increased in patients with atherosclerosis, hypertension or with diabetes mellitus and that sEng can be considered as a biomarker of endothelial damage. ${ }^{2}$

Endoglin (transforming growth factor- $\beta$ receptor III or CD 105) is a homodimeric transmembrane glycoprotein, which belongs to the family of zona 
pellucida of extracellular proteins. It is an accessory receptor for TGF- $\beta$ (transforming growth factor $-\beta$ ) superfamily and it regulates several cellular processes, such as: proliferation, differentiation, adhesion and migration. ${ }^{4}$

Two forms of CD105 are currently studied: a) a membrane form, expressed in various tissues and studied in some cardiovascular pathologies (atherosclerosis, preeclampsia, telangiectasia, hereditary hemorrhagia) and cancer and b) a soluble form (sEng), found in high concentrations in plasma after endothelial injury. sEng is expressed by the endothelial cells and has a crucial role in the functioning and homeostasis of the endothelium. ${ }^{5}$ Low concentrations of sEng were found in normal endothelial cells, being highly expressed in: inflamed endothelial cells, ${ }^{1}$ vascular injuries, ${ }^{6}$ tumor vessels, ${ }^{7}$ during embryogenesis, ${ }^{8}$ angiogenesis, ${ }^{9}$ vascular smooth muscle cells of atherosclerotic plaque, ${ }^{10}$ fibroblasts, activated monocytes and macrophages. ${ }^{4}$

A hypertensive effect of high levels of sEng was shown in animal models and in patients with preeclampsia, sEng representing a possible disease severity biomarker, due to its interference with TGF- $\beta /$ TGF- $\beta$ pathway receptor and thus inhibiting the eNOS (endothelial nitric oxide synthase) -dependent vasodilatation. ${ }^{11,12}$ As well as other studies revealed that sEng increased the expression of P-selectin, E-selectin, soluble VCAM-1 and ICAM-1 on endothelium. ${ }^{11,12}$

Considering all the information presented above and also suggested by Rathouska J et al 2015, sEng might be an interesting biomarker to be studied in cardiovascular pathologies induced by ED or hypercholesterolemia under different regimens of treatment, as a reflection of the efficacy of the treatment. ${ }^{2}$

Accordingly, this study aimed to quantify sEng plasma levels in hypertensive patients with endothelial dysfunction under different antihypertensive regimens of treatment.

\section{Material and methods}

A cross-sectional, comparative study was conducted from January 2015 to September 2016 in the Cardiology Clinic of Timisoara City Hospital, Romania.

A total of 323 patients were enrolled in the present study, from which: 99 patients with normal values of blood pressure (Group A - control group), 106 hypertensive patients treated with different types of antihypertensive molecules (beta blockers: metoprolol 50 or $100 \mathrm{mg} / \mathrm{day}$, carvedilol 12.5 or $25 \mathrm{mg} /$ day, nebivolol $5 \mathrm{mg} /$ day, bisoprolol 5 or $10 \mathrm{mg} /$ day; calcium channel blockers: amlodipine 5 or $10 \mathrm{mg} /$ day, lercanidipine 10 or $20 \mathrm{mg}$ /day and diuretics: indapamide 1.5 or $2.5 \mathrm{mg} /$ day, furosemide $20 \mathrm{mg}+$ spironolactone $50 \mathrm{mg} /$ day), in monotherapy (Group B) and 118 hypertensive patients treated with perindopril (5 or $10 \mathrm{mg}$ / day), in monotherapy (Group C). All the patients were matched by age and sex.

\section{Patient selection}

All recruited patients completed the informed consent form and participated voluntary in the study.

The patients enrolled in the control group were initially examined for the suspicion of essential arterial hypertension, but the $24 \mathrm{hrs}$ monitoring device did not confirm the diagnosis.

The patients enrolled in Group B were under chronic treatment with other types of antihypertensive molecules (beta blockers, calcium channels blockers, diuretics), in monotherapy.

And the patients enrolled in Group $\mathrm{C}$ were under chronic treatment with perindopril 5 or $10 \mathrm{mg} /$ day, in monotherapy as well.

The hypertensive patients were enrolled based on: age $>18$ years, diagnosis of essential arterial hypertension for at least one year (values $>140 / 90 \mathrm{mmHg}$ ) and monotherapy with one of the drugs/classes mentioned above. Patients with other pathologies such as: coronary artery disease, atherosclerotic disease (carotid or peripheral), diabetes, heart failure, hepatic and/or renal diseases, acute or chronic inflammatory pathologies were excluded from the study. Moreover, patients treated with other angiotensin-converting enzyme (ACE) inhibitors or angiotensin receptor blockers (ARBs) were excluded from this study due to their similarity in reversing the endothelial dysfunction and that the results could be more accurate and precise.

All recruited patients underwent a screening including the pathology and other associated medical conditions record, familial medical history, laboratory and paraclinical investigations.

\section{Laboratory analysis}

All recruited patients were examined in the morning, in a temperature controlled room and were in a fasting state $>12$ h. Standard biochemical analysis such as: total cholesterol, triglycerides, serum glucose etc were performed inside the hospital, by routine laboratory methods, after the venous blood samples had been collected, prior to minimum 20 mins of rest. sEng, pentraxin-3 (PTX3) and high 
sensitivity $\mathrm{C}$ reactive protein (hs-CRP) plasma levels were assessed by the Bioclinica SA Laboratory from Timisoara, Romania. sEng and PTX3 plasma levels were determined with the quantitative sandwich enzyme immunoassay technique distributed by the R\&D Systems. hs-CRP was measured using a CRP Ultra Kit, through a highly sensitive immunoturbidimetric method, distributed by Abbott Diagnostics. All the methods being standardized, we considered that there was no need to repeat them here.

\section{Arterial pressure}

The blood pressure was measured in the same morning, at the right brachial artery, in a temperature controlled room, after minimum 20 mins of rest, in a supine position. It was expressed as the mean value of 3 measurements.

The presence or absence of the ED was assessed in two ways: through a functional assessment - by the flow mediated dilation measurement dependent of the endothelium (FMD) and through a structural assessment - the intima-media thickness (IMT).

\section{Assessment of flow-mediated vasodilatation (FMD)}

The procedure was performed after the patient stopped the vasoactive medication for at least $10 \mathrm{hrs,} \mathrm{with} \mathrm{a} \mathrm{fasting}$ and no smoking period of $12 \mathrm{hrs}$ and after 20 mins of rest in a quiet room. After the patient was positioned in a supine posture with the arm in a comfortable position, the brachial artery was imaged above the antecubital fossa in the longitudinal plane using a linear transducer with $9 \mathrm{MHz}$. The dimension of the brachial artery was measured manually with an electronic calliper. The ischemia was produced by inflating the cuff of a manometer (placed at the distal forearm) with a pressure of $50 \mathrm{mmHg}$ greater than the systolic blood pressure of the patient. The release of the cuff after 5 mins generated an increased diameter of the brachial artery, the maximal diameter being measured $1 \mathrm{~min}$ after cuff release. The diameter of the artery was being measured at the same moment of the cardiac cycle.

FMD is the percent of diameter change from rest to $1 \mathrm{~min}$ after ischemia and we considered it normal at a $20 \%$ increase.

\section{Carotid intima-media thickness assessment (IMT)}

This assessment was done in B-mode ultrasonography with a GE medical system VIVID S5, high-resolution ultrasonography system, equipped with a $9-\mathrm{MHz}$ linear array transducer. The measurements were done on the distal $1 \mathrm{~cm}$ common carotid artery at both sides. Three determinations were done on each side and the arithmetical mean of the value was registered. An increased IMT was considered when IMT $>0.9 \mathrm{~mm}$ and a plaque if there was a localized thickening $>1.5 \mathrm{~mm}$.

\section{Echocardiography}

Echocardiographic measurements were performed by a General Electric medical system VIVID S5, highresolution ultrasonography system. We assessed the consequence of hypertension over the heart structures and functions. From beginning we excluded the patients with low ejection fraction ( $\mathrm{EF}<40 \%$ ). The following parameters were recorded: the diameter of left atrium (DLA), the dimension of the interventricular septum (IVS), posterior wall of the left ventricle (PWLV), ejection fraction (EF) and theend diastolic diameter of the left ventricle (EDLV).

All the measurements were performed by the same certified cardiologist.

\section{Statistical analyses}

Numerical data are presented as mean \pm standard deviation (SD) and median (25-75th). The statistical software used was SPSS v.17. A statistical significance was considered at a $P$-value $<0.05$. Categorical data are presented as frequency (\%). ANOVA analysis and Kruskal-Wallis tests were performed in order to obtain the differences among the groups and the Spearman's correlation was used to obtain the correlations between different variables.

\section{Results}

A total of 323 patients were enrolled in this study, from which 99 were non-hypertensive patients, representing the control group, 53 men and 46 women, with the mean age of $54.62 \pm 14.64$.

The rest of 224 patients were enrolled in the study group as follows: 106 hypertensive patients under chronic treatment with other antihypertensive drugs, representing Group B - 44 men and 62 women the mean age being $62.11 \pm 13.11$ and 118 hypertensive patients under chronic treatment with perindopril, representing Group $\mathrm{C}-55$ men and 63 women, the mean age being $61.07 \pm 12.64$ (Table 1, part I).

It can be noticed that both hypertensive groups (Group $\mathrm{B}$ and $\mathrm{C}$ ) have controlled blood pressure levels under the chronic antihypertensive treatment, with similar history 
Table I Patients' characteristics

\begin{tabular}{|c|c|c|c|c|}
\hline Parameters & $\begin{array}{l}\text { Group A - Control } \\
(n=99)\end{array}$ & $\begin{array}{l}\text { Group B - other anti-HTN } \\
(n=106)\end{array}$ & $\begin{array}{l}\text { Group C - perindopril } \\
(n=\mid 18)\end{array}$ & $p$ \\
\hline \multicolumn{5}{|c|}{ I. Demographic and clinical characteristics } \\
\hline Age (years) & $54.62 \pm 14.64$ & $62.11 \pm 13.11(*)$ & $61.07 \pm 12.64(*)$ & $<0.0001$ \\
\hline Men (total) & $53(99)$ & $44(106)$ & $55(118)$ & - \\
\hline$\%$ & $53.53 \%$ & $41.50 \%$ & $46.61 \%$ & - \\
\hline Women (total) & $46(99)$ & $62(106)$ & $63(118)$ & - \\
\hline$\%$ & $46.46 \%$ & $58.49 \%$ & $53.38 \%$ & - \\
\hline SBP $(\mathbf{m m H g})$ & $|22.26 \pm| 7.7 \mid$ & $139.65 \pm 18.89(*)$ & $139.63 \pm 15.80(*)$ & $<0.0001$ \\
\hline DBP (mmHg) & $76.52 \pm 9.80$ & $83.26 \pm 13.50(*)$ & $84.44 \pm 10.69(*)$ & $<0.0001$ \\
\hline HTN duration (months) & & $103.92 \pm 82.93$ & $102.56 \pm 77.00$ & 0.89 \\
\hline Heart rate (beats/min) & $70.21 \pm 8.67$ & $75.49 \pm 10.78(\#)$ & $69.31 \pm 7.01(\#)$ & $<0.0001$ \\
\hline \multicolumn{5}{|c|}{ II. Indicators of the presence/absence of endothelial dysfunction } \\
\hline Soluble endoglin (ng/mL) & $5.49 \pm 3.00$ & $5.63 \pm 2.33(\#)$ & $4.73 \pm 1.39(\#)$ & $<0.01$ \\
\hline Pentraxin-3 (ng/mL) & $1.41 \pm 1.37$ & $1.27 \pm 1.20(\#)$ & $0.86 \pm 0.56(\#)$ & $<0.001$ \\
\hline hs-CRP (mg/dL) & $0.26 \pm 0.03$ & $0.26 \pm 0.02(\#)$ & $0.38 \pm 0.53(\#)$ & 0.02 \\
\hline ED (presence) & $35(99)$ & $76(106)$ & $76(118)$ & - \\
\hline$\%$ & $35.35 \%$ & $71.69 \%$ & $64.40 \%$ & - \\
\hline FMD (\%) & $19.51 \pm 0.06$ & $10.41 \pm 0.06(\#, *)$ & $11.36 \pm 0.06(\#, *)$ & $<0.001$ \\
\hline CC IMT right (mm) & $0.84 \pm 0.24$ & $0.92 \pm 0.19(\#, *)$ & $0.95 \pm 0.21 \quad(\#, *)$ & $<0.001$ \\
\hline CC IMT left (mm) & $0.83 \pm 0.20$ & $0.95 \pm 0.18(\#, *)$ & $0.92 \pm 0.18(\#, *)$ & $<0.001$ \\
\hline Smokers (total) & $19(99)$ & $16(106)$ & $30(118)$ & - \\
\hline$\%$ & $19.19 \%$ & $15.09 \%$ & $25.42 \%$ & - \\
\hline \multicolumn{5}{|c|}{ III. Biochemical characteristics } \\
\hline Glucose (mg/dL) & $110.63 \pm 52.08$ & $110.83 \pm 42.30$ & $109.06 \pm 19.52$ & 0.93 \\
\hline Cholesterol (mg/dL) & $200.12 \pm 56.10$ & $204.11 \pm 51.69(\#)$ & $217.58 \pm 58.64(\#)$ & 0.05 \\
\hline Triglicerides (mg/dL) & $125.29 \pm 73.67$ & $140.40 \pm 17.30$ & $\mid 35.87 \pm 58.36$ & 0.61 \\
\hline Creatinine $(\mathrm{mg} / \mathrm{dL})$ & $0.85 \pm 0.22$ & $0.86 \pm 0.20(\#)$ & $0.97 \pm 0.32(\#)$ & $<0.0001$ \\
\hline Potassium (mmol/L) & $4.36 \pm 0.38$ & $4.24 \pm 0.47(\#)$ & $4.40 \pm 0.46(\#)$ & 0.01 \\
\hline INR & $1.17 \pm 0.33$ & $1.05 \pm 0.22$ & $1.32 \pm 0.14$ & 0.08 \\
\hline ALAT (U/L) & $39.37 \pm 15.86$ & $40.02 \pm 14.47$ & $43.70 \pm 16.46$ & 0.09 \\
\hline ASAT (U/L) & $24.79 \pm 9.22$ & $23.67 \pm 7.96$ & $25.96 \pm 11.12$ & 0.20 \\
\hline $\operatorname{ESR}(\mathrm{mm} / \mathrm{h})$ & $9.35 \pm 5.56$ & $13.19 \pm 7.87(\#, *)$ & $16.28 \pm 12.65(\#, *)$ & $<0.0001$ \\
\hline BUN (mg/dL) & $18.64 \pm 6.53$ & $17.63 \pm 7.05$ & $18.89 \pm 6.69$ & 0.35 \\
\hline BMI $\left(\mathrm{kg} / \mathrm{m}^{2}\right)$ & $23.91 \pm 3.74$ & $26.34 \pm 3.8 \mathrm{I}(\#, *)$ & $28.06 \pm 3.24(\#, *)$ & $<0.0001$ \\
\hline Fibrinogen (mg/dL) & $314.00 \pm 54.37$ & $316.03 \pm 49.90(\#)$ & $295.67 \pm 54.29(\#)$ & $<0.01$ \\
\hline \multicolumn{5}{|c|}{ IV. Echocardiographic characteristics } \\
\hline DLA (mm) & $34.99 \pm 7.01$ & $37.38 \pm 7.04(*)$ & $36.47 \pm 5.65\left(^{*}\right)$ & 0.03 \\
\hline IVS (mm) & $10.77 \pm 2.57$ & $11.34 \pm 2.32$ & $11.20 \pm 1.79$ & 0.17 \\
\hline PWLV (mm) & $10.01 \pm 1.63$ & $10.46 \pm 1.84(\#, *)$ & $10.85 \pm 1.51 \quad(\#, *)$ & $<0.01$ \\
\hline EDLV (mm) & $50.08 \pm 6.64$ & $52.73 \pm 5.44(\#)$ & $50.94 \pm 4.85(\#)$ & $<0.001$ \\
\hline E.F. (\%) & $56.08 \pm 8.33$ & $52.74 \pm 8.81$ (\#) & $55.57 \pm 7.90(\#)$ & $<0.01$ \\
\hline
\end{tabular}

Notes: Data are represented as mean \pm standard deviation (SD). Statistical significance was considered at a $\mathrm{p}$-value $<0.05$. $(*)$ : Significant difference when compared with the control group. (\#): Significant difference between both hypertensive groups.

Abbreviations: SBP, systolic blood pressure; DBP, diastolic blood pressure, HTN, essential arterial hypertension; hs-CRP, high-sensitivity C-reactive protein; PTX3, pentraxin-3; ED, endothelial dysfunction; FMD, flow mediated vasodilatation; CC IMT, common carotid intima-media thickness; INR, international normalized ratio; ALAT, alanine aminotransferase (GTP); ASAT, aspartate aminotransferase (GOT); ESR, erythrocyte sedimentation rate; BUN, blood urea nitrogen; BMI, body mass index; DLA, diameter of left atrium; IVS, diameter of interventricular septum; PWLV, posterior wall of the left ventricle; EDLV, end diastolic diameter of left ventricle; EF, ejection fraction. 
period of hypertension. Also both groups were similar in the terms of age, sex distribution and blood pressure levels. The heart rate was lower in Group $\mathrm{C}$ compared with Group B, $p<0.0001$ (Table 1, part I).

Regarding the indicators of the presence/absence of ED (part II of the Table 1): both biochemical markers (endoglin and pentraxin-3) have decreased plasma levels in the group of patients treated with perindopril (Group C) compared with Group B and Group A, the difference being statistically significant. Group B of patients (under chronic treatment with other antihypertensive regimens) having the highest sEng plasma levels of all. The functional and structural parameters (FMD and IMT) are similar in both groups of hypertensive patients, compared with the control group, the difference being statistically significant as well (Group A versus Group B and C). The percent of flow mediated vasodilatation is higher in the group of patients under chronic treatment with perindopril (Group C) compared with the percent from Group B. Moreover Group $\mathrm{C}$ has a lower number of patients with $\mathrm{ED}$, as well as a lower plasma level of sEng and PTX3, although this group has the highest percent of smokers.

With respect to the biochemical data presented in section III of the Table 1, it can be noticed differences (statistically significant) between the two hypertensive groups compared with the normotensive group (control group) of patients, with the level of several parameters such as: cholesterol, triglycerides, transaminases being higher in Group C and B, than in Group A. The creatinine and potassium levels were higher in Group C, as expected, due to the mechanism of action of the ACE inhibitor, perindopril. Erythrocyte sedimentation rate (ESR) plasma levels were higher in group $\mathrm{C}$ as a possible consequence of a systemic inflammation installed, reflected also through
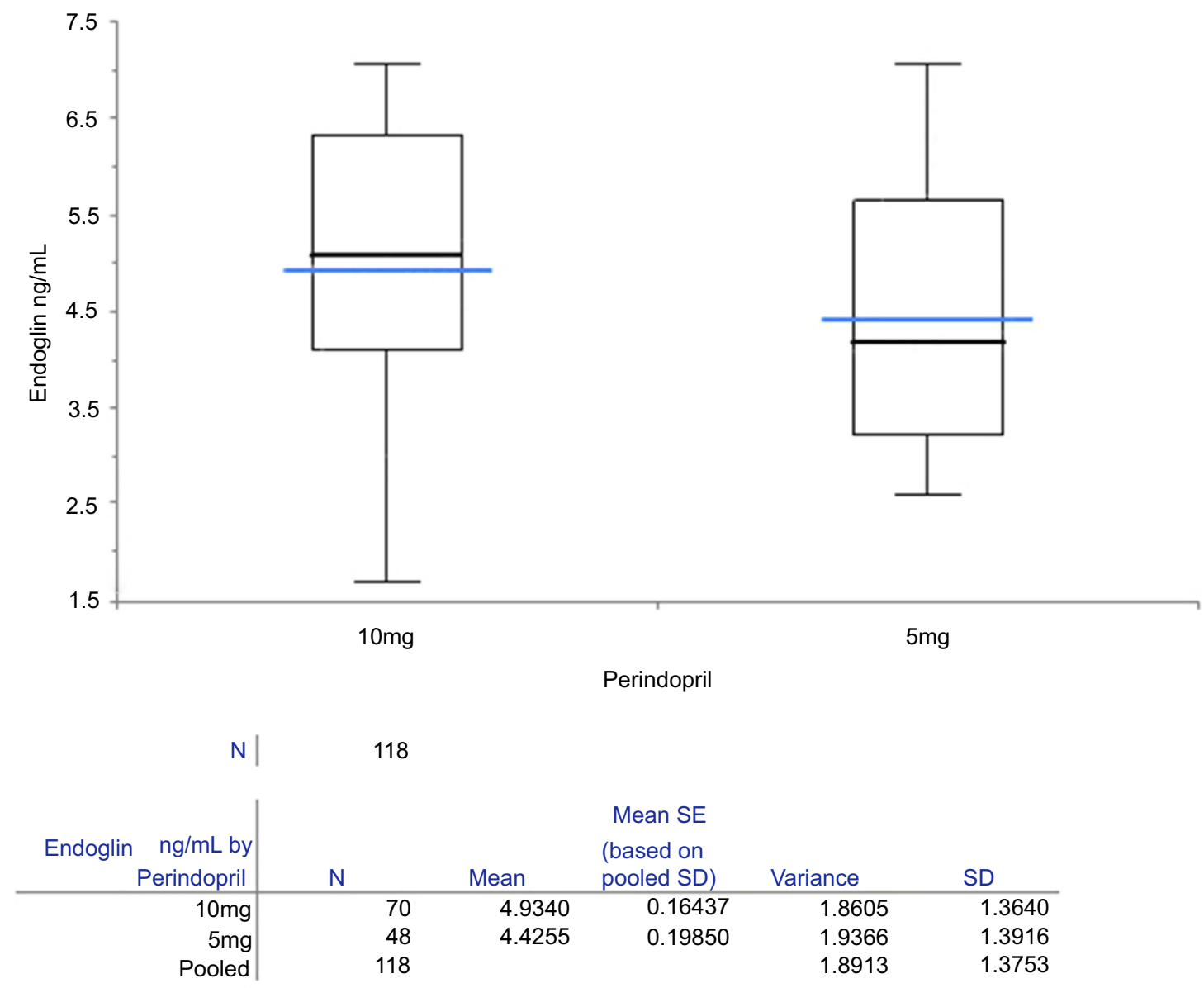

\begin{tabular}{r|rrrrrr}
$\begin{array}{r}\text { Endoglin } \mathrm{ng} / \mathrm{mL} \text { by } \\
\text { Perindopril }\end{array}$ & \multicolumn{1}{c}{ Minimum } & 1st quartile & \multicolumn{1}{c}{ Median } & 3rd quartile & Maximum & $\begin{array}{c}\text { Inter-quartile } \\
\text { range }\end{array}$ \\
\hline $10 \mathrm{mg}$ & 1.713 & 4.1074 & 5.1050 & 6.3287 & 7.069 & 2.2213 \\
$5 \mathrm{mg}$ & 2.615 & 3.2324 & 4.2035 & 5.6500 & 7.060 & 2.4176
\end{tabular}

Figure I The mean soluble endoglin plasma levels in function of the concentration of perindopril. 

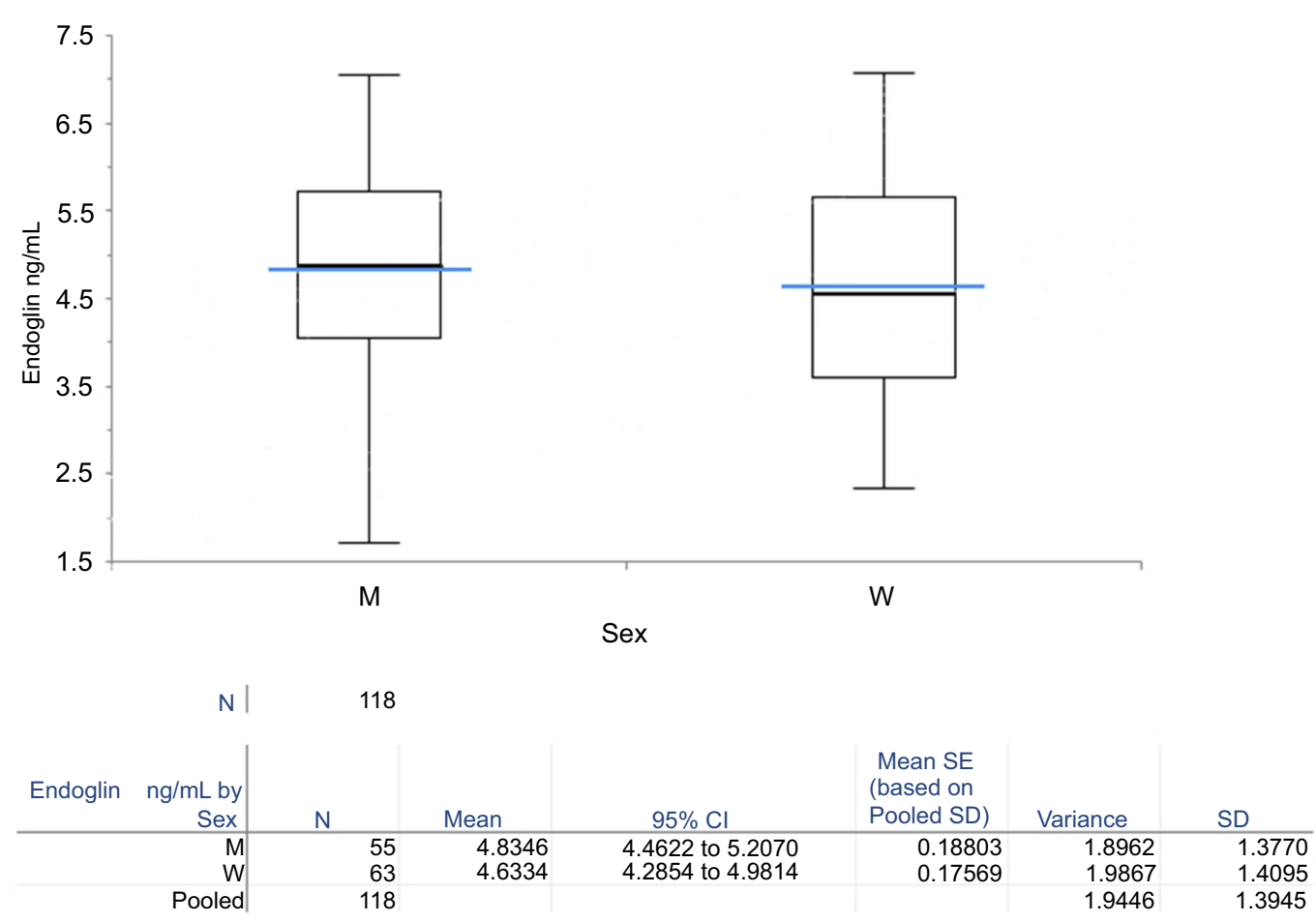

\begin{tabular}{|c|c|c|c|c|c|c|c|}
\hline 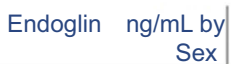 & Minimum & 1st quartile & Median & $95 \% \mathrm{Cl}$ & 3rd quartile & Maximum & $\begin{array}{l}\text { Inter-quartile } \\
\text { range }\end{array}$ \\
\hline $\mathrm{M}$ & 1.713 & 4.0453 & 4.8700 & 4.2010 to 5.2110 & 5.7333 & 7.060 & 1.6880 \\
\hline W & 2.346 & 3.6122 & 4.5530 & 3.8950 to 5.1200 & 5.6623 & 7.069 & 2.0502 \\
\hline
\end{tabular}

Figure 2 Soluble endoglin plasma levels in Group C, as a function of patients' sex. Abbreviations: M, men; W, women.

the hs-CRP levels, although the fibrinogen plasma level was lower. Moreover, body mass index (BMI) was higher in Group C.

The section IV of Table 1 presents the echocardiography parameters of the studied patients. In Group B it can be noticed a dilatation of the left ventricle, reflected through the end diastolic diameter of left ventricle with a lower ejection fraction, statistically significant compared with the other groups. Regarding the duration of hypertension and its consequences over the heart structures, no statistical difference can be observed between the control group (Group A) and the group of patients treated with perindopril (Group C) in terms of echocardiography parameters.

There were no correlations found between endoglin and the rest of the parameters in gr. C, as well as in gr. B.

From Figure 1 it can be seen that in Group C, patients under chronic treatment with $5 \mathrm{mg}$ /day of perindopril had lower endoglin plasma levels compared with patients receiving $10 \mathrm{mg} / \mathrm{day}$.

In the patients from Group C, women had lower plasma levels of sEng compared with men, although the values are very closely related (4.63 versus 4.83$)$ Figure 2.

The mean plasma level of sEng was higher in Group $\mathrm{B}-(5.63 \pm 2.33)$ as follows: beta blockers $-5.93 \pm 2.54$ vs $4.73 \pm 1.39, p<0.0001$, calcium channel blockers -5.74 \pm 2.14 vs $4.73 \pm 1.39, p<0.001$ and diuretics $5.15 \pm 2.24$ vs $4.73 \pm 1.39, p=0.164$ - Figures 3-5; Table 2), compared with Group C $-4.73 \pm 1.39, p<0.01$ (Figures 1 and 2). The highest sEng plasma level was found in patients under chronic treatment with beta blockers (Figure 3, Table 2).

\section{Discussion}

Perindopril is a 3rd generation ACE inhibitor, a first line agent prescribed for the treatment of several pathologies such as: essential arterial hypertension, heart failure, myocardial infarction, systolic or diastolic dysfunction of left ventricle. As well as other ACE inhibitors, it blocks the converting enzyme of angiotensin I (Ang I) in the endothelial cells of the lungs, other organs and blood (to a lower level), inducing a diminution of angiotensin II (Ang II) plasma levels. This will later increase Ang I plasma levels and through a negative feed-back phenomenon it will 

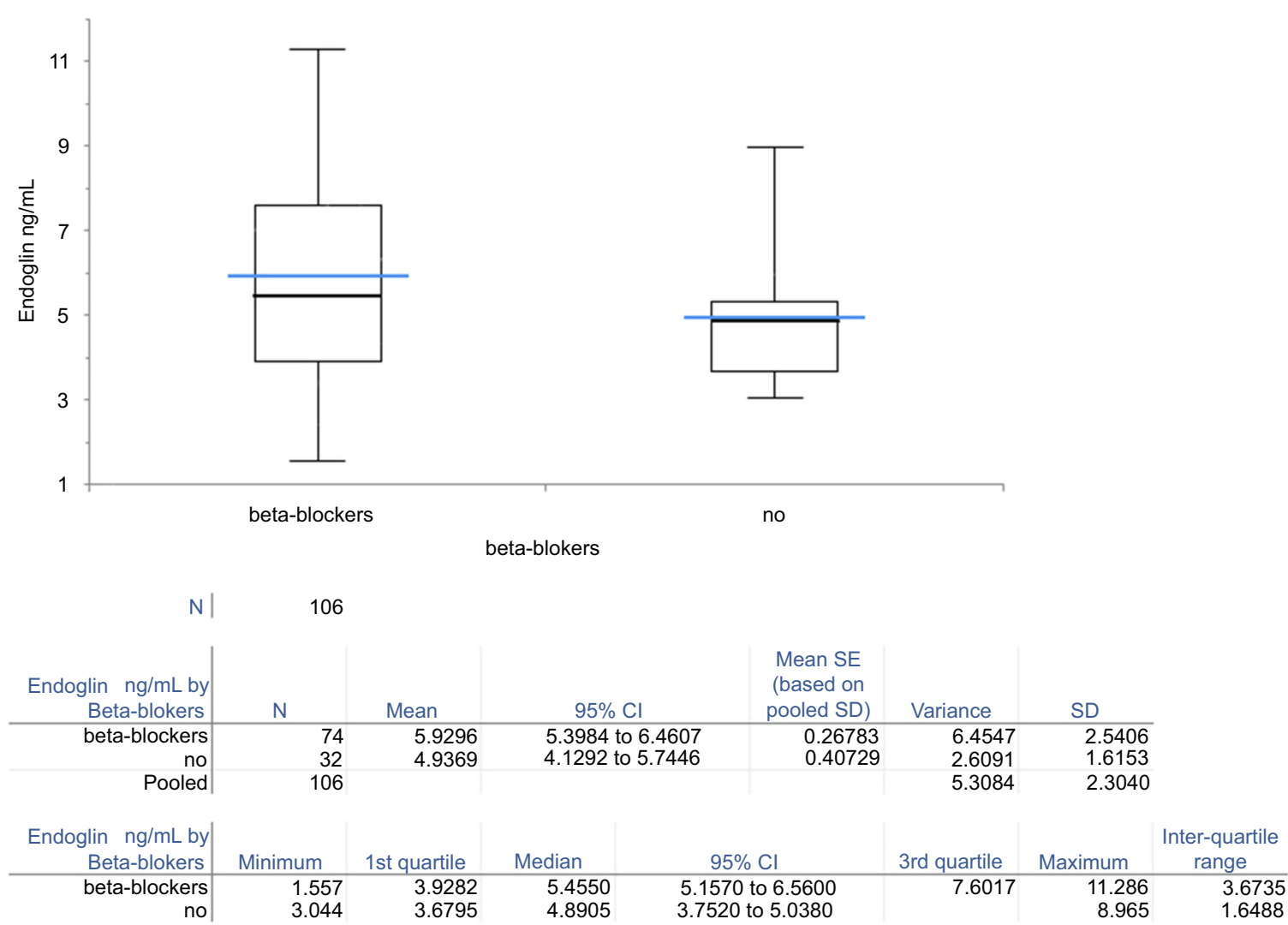

Figure 3 Soluble endoglin plasma levels in Group B, in patients treated with beta blockers.

stimulate the production of renin, lowering the angiotensinogen concentrations (due to the increased consumption). ${ }^{13}$ The decreasing of Ang II plasma levels has several consequences such as: a lower aldosterone secretion, modifications in the sodium-potassium balance, a lower action on the endothelial tonus etc. Moreover, because ACE is identical with kinase II - the enzyme that degrades bradykinin, perindopril increases the bradykinins' concentrations, mediating also their endogenous actions: nitric oxide (NO), prostaglandins, endothelial derived hyperpolarizing factor (EDHF) secretion, by stimulating $\mathrm{B}_{2}$ receptors of bradykinin. Thus, perindopril, as well as other ACE inhibitors, possess a vascular protective action, reversing the endothelial dysfunction. ${ }^{14}$ Perindopril also induces renal protection, it decreases microalbuminuria and can ameliorate insulin resistance in diabetic patients. $^{15}$

Taking into account perindopril's properties of reversing endothelial dysfunction and the fact that sEng was proposed as a marker of endothelial damage, we aimed to evaluate perindopril's impact on sEng plasma levels in comparison with other classes of antihypertensive drugs, administered as a chronic treatment. ${ }^{2,16}$
sEng is defined as an extracellular domain of the entire endoglin membrane which enters the systemic circulation in case of endothelial injury, inflammation, activation and senescence. It is highly expressed in endothelial cells, having an important role in vascular homeostasis. Moreover, it has been detected in plasma, serum and urine of patients with alterations of angiogenesis, sEng possessing anti-angiogenic properties, compared with the membrane endoglin. Several studies performed in the cardiovascular field showed a connection between increased sEng levels and different pathologies linked to endothelial dysfunction . $^{2}$

sEng plasma levels were reported to be increased after treatment with tumor necrosis factor (TNF)- $\alpha$, the inflammatory cytokine, and after $\mathrm{H}_{2} \mathrm{O}_{2}$ induction of oxidative stress (a key point of endothelial dysfunction). In contrary, HO-1, a vascular protective substance, was found to inhibit sEng release form endothelial cells. ${ }^{17}$ Blann et al, 1996, was the first to show increased levels of sEng in hypercholesterolemic patients and Li et al, 2000, reported high sEng levels in the early stages of atherosclerotic process as a consequence of the damaged endothelial cells. ${ }^{5,18}$ In another study, diabetic patients who associated or not essential arterial 

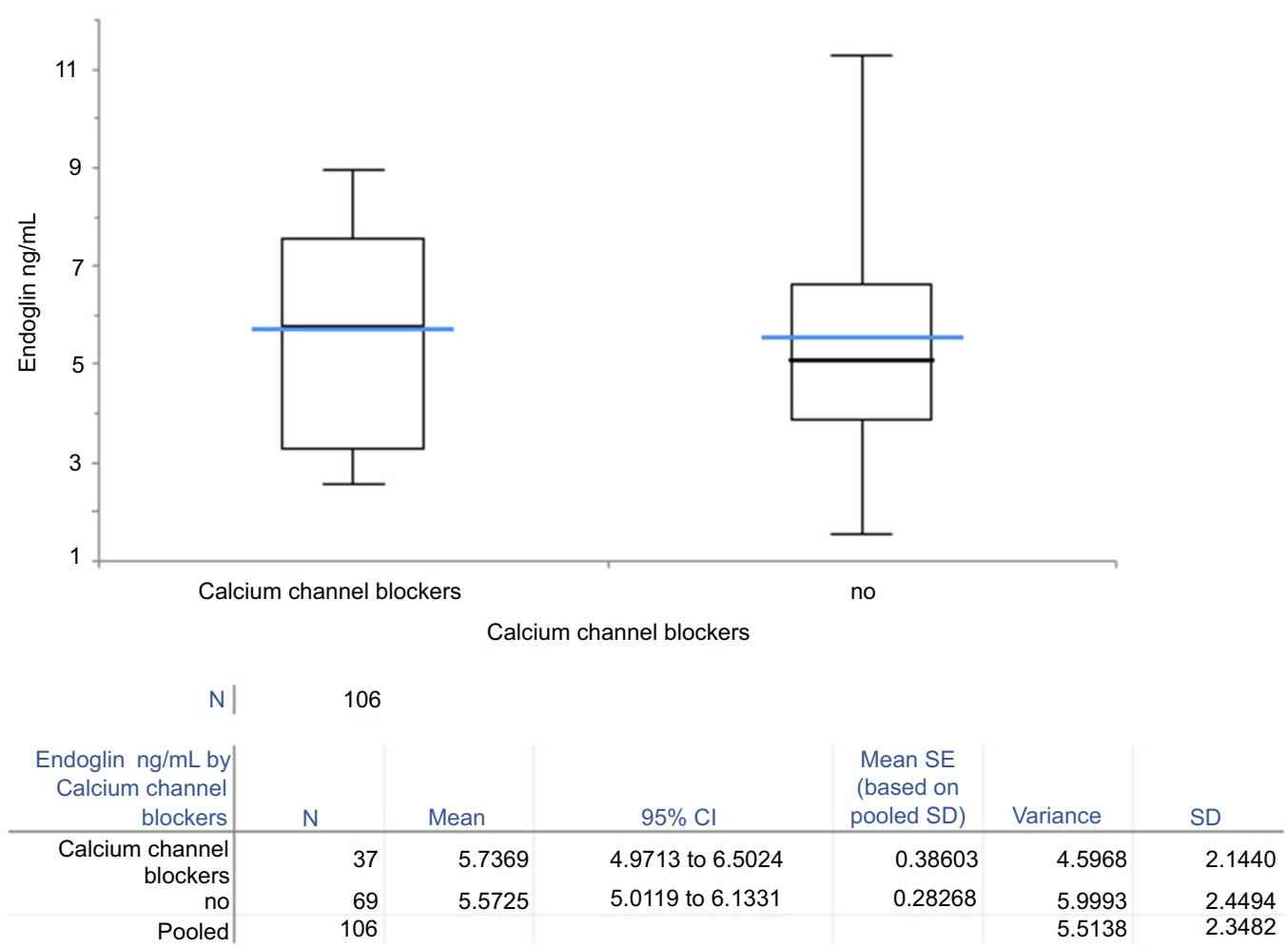

\begin{tabular}{|c|c|c|c|c|c|c|c|}
\hline $\begin{array}{r}\text { Endoglin } \mathrm{ng} / \mathrm{mL} \text { by } \\
\text { Calcium channel } \\
\text { blockers }\end{array}$ & Minimum & 1st quartile & Median & $95 \% \mathrm{Cl}$ & 3rd quartile & Maximum & $\begin{array}{l}\text { Inter-quartile } \\
\text { range }\end{array}$ \\
\hline Calcium channel & 2.577 & 3.3093 & 5.7840 & 4.8400 to 6.9880 & 7.5840 & 8.965 & 4.2747 \\
\hline 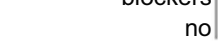 & 1.557 & 3.8813 & 5.1020 & 4.2520 to 5.4540 & 6.6183 & 11.286 & 2.7370 \\
\hline
\end{tabular}

Figure 4 Soluble endoglin plasma levels in Group B, in patients treated with calcium channel blockers.

hypertension had higher sEng levels compared with the control group and in patients with coronary artery disease sEng levels had been directly linked to the vascular damage. ${ }^{1,17}$

In our study, the highest sEng plasma level was found in the group of patients under chronic treatment with other classes of antihypertensive drugs - Group B, the highest mean value for this group being recorded for patients treated with beta blockers $-5.93 \mathrm{ng} / \mathrm{mL}$ (Figure 3, Table 2). The lowest sEng plasma levels were found in Group C of patients under chronic treatment with perindopril (Table 1, Figure 2). Moreover, Pentraxin-3 plasma levels (other marker of endothelial damage and acute inflammation ${ }^{19}$ ) were the lowest in this group (Group C) suggesting a better control of the endothelial function and inflammation in patients under chronic treatment with perindopril. This aspect is sustained by other studies which revealed high sEng levels in inflamed tissues and healing wounds. ${ }^{20}$

Moreover, the improved endothelial function in Group C (compared with Group B) is also sustained by FMD level $(11.36 \%$ versus $10.41 \%)$, although
Group $\mathrm{C}$ has the highest percent of smokers: $25.42 \%$ versus $15.09 \%$.

It is important to mention that both hypertensive groups (B and C) had controlled values of blood pressure under different antihypertensive regimens of treatment, with similar history period of hypertension, although the heart rate was higher in Group B.

Regarding the echocardiography parameters, previous studies reported high levels of circulating sEng in patients with suspected left ventricular dysfunction, which were strongly correlated with elevated left ventricular enddiastolic pressure, reduced ejection fraction, all of them being considered predictors of mortality in heart failure patients. ${ }^{21}$ Aspect sustained by our study as well, as high sEng plasma levels were found in Group B where patients had a higher end diastolic diameter of left ventricle and a lower ejection fraction, compared with Group C.

Also reduced endoglin activity has been shown to regulate cardiac remodeling in heart failure and myocardial infarction. ${ }^{22}$ 

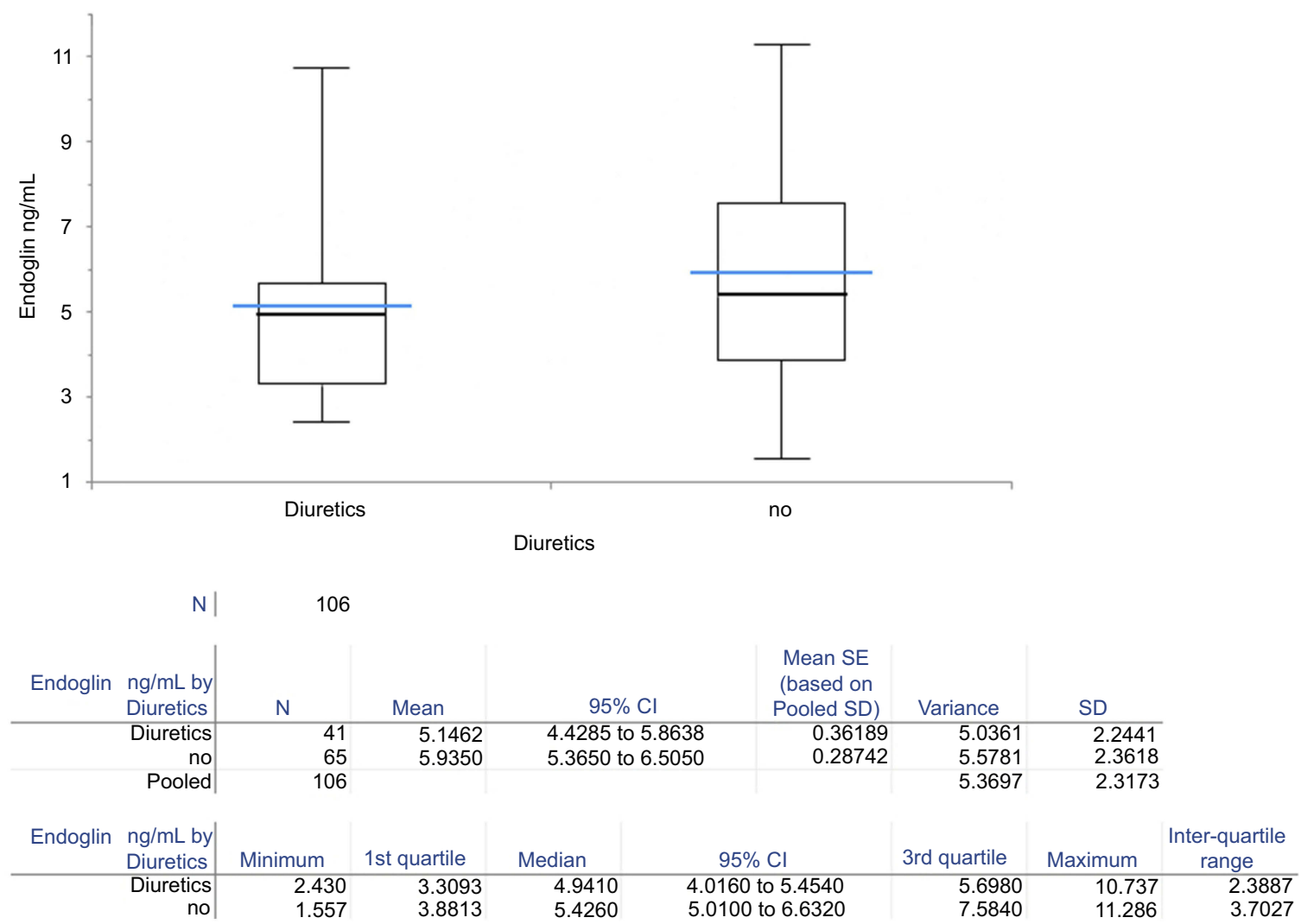

Figure 5 Soluble endoglin plasma levels in Group B, in patients treated with diuretics.

Table 2 Soluble endoglin plasma levels in both antyhypertensive groups (Group B and C)

\begin{tabular}{|l|l|l|l|}
\hline & Soluble endoglin in Group B (ng/mL) & Soluble endoglin in Group C (ng/mL) & $\mathbf{P}$ \\
\hline Beta blockers & $5.93 \pm 2.54$ & $4.73 \pm 1.39$ & $<0.0001$ \\
Calcium channel blockers & $5.74 \pm 2.14$ & $4.73 \pm 1.39$ & $<0.001$ \\
Diuretics & $5.15 \pm 2.24$ & $4.73 \pm 1.39$ & 0.164 \\
\hline
\end{tabular}

In animal models, trandolapril was found to decrease renal fibrosis based on a reduction in TGF-betal expression, although it did not reduce increased endoglin expression. ${ }^{23}$ Other study showed that hypolipemiant treatment with atorvastatin can reduce not only cholesterol levels and the plaque size, but also the sEng plasma levels, increasing its membrane expression in aorta. ${ }^{24}$

Our study has several limitations like: relatively low number of patients examined and the fact that it does not explain the molecular mechanisms by which perindopril lowers the sEng plasma levels in hypertensive patients with ED.

\section{Conclusion}

Decreased sEng plasma levels were found in patients under chronic treatment with perindopril compared with other regimens of chronic treatment (beta blockers, calcium channel blockers and diuretics). Further studies regarding sEng plasma levels in patients treated with ARBs are needed in order to have a more complete image of the influence of classical antihypertensive treatment on sEng plasma levels in hypertensive patients with endothelial dysfunction.

\section{Compliance with the ethical standards}

The present study has been approved by the Ethical Committee of the "Victor Babes" University of Medicine and Pharmacy, Timisoara, Romania, no. 7/2016. All procedures performed in this study with human participants were in accordance with the ethical standards of the institutional research committee and with the 1964 Helsinki declaration and its later amendments or comparable ethical standards. 


\section{Acknowledgments}

This work was supported by the grant PII-C4-TC-2016/ 16441-09 offered by the "Victor Babes" University of Medicine and Pharmacy of Timisoara, Romania.

\section{Author contributions}

All authors made substantial contributions to conception, design, data acquisition, analysis and interpretation. All authors took part in drafting the article and revising it critically for important intellectual content. Also, they gave final approval of the version to be published and agree to be accountable for all aspects of the work.

\section{Disclosure}

The authors report no conflicts of interest in this work.

\section{References}

1. Blázquez-Medela AM, García-Ortiz L, Gómez-Marcos MA, et al. Increased plasma soluble endoglin levels as an indicator of cardiovascular alterations in hypertensive and diabetic patients. BMC Med. 2010;8:86. doi:10.1186/1741-7015-8-86

2. Rathouska J, Jezkova K, Nemeckova I, Nachtigal P. Soluble endoglin, hypercholesterolemia and endothelial dysfunction. Atherosclerosis. 2015;243(2):383-388. doi:10.1016/j.atherosclerosis.2015.10.003

3. Davignon J. Cardioprotective and other emerging effects of statins. Int J Clin Pract Suppl. 2004;58(143):49-57.

4. Rakocevic J, Orlic D, Mitrovic-Ajtic O, et al. Endothelial cell markers from clinician's perspective. Exp Mol Pathol. 2017;102(2):303-313. doi:10.1016/j.yexmp.2017.02.005

5. Blann AD, Wang JM, Wilson PB, Kumar S. Serum levels of the TGF-beta receptor are increased in atherosclerosis. Atherosclerosis. 1996;120(1-2):221-226.

6. Botella LM, Sánchez-Elsner T, Sanz-Rodriguez $\mathrm{F}$, et al. Transcriptional activation of endoglin and transforming growth factor-beta signaling components by cooperative interaction between Sp1 and KLF6: their potential role in the response to vascular injury. Blood. 2002;100(12):4001-4010. doi:10.1182/blood.V100.12.4001

7. Miller DW, Graulich W, Karges B, et al. Elevated expression of endoglin, a component of the TGF-beta-receptor complex, correlates with proliferation of tumor endothelial cells. Int J Cancer. 1999;81(4):568-572.

8. Perlingeiro RC. Endoglin is required for hemangioblast and early hematopoietic development. Development. 2007;134(16):3041-3048. doi: 10.1242/dev.002907

9. Bernabeu C, Lopez-Novoa JM, Quintanilla M. The emerging role of TGF-beta superfamily coreceptors in cancer. Biochim Biophys Acta. 2009;1792(10):954-973. doi:10.1016/j.bbadis.2009.07.003
10. Conley BA, Smith JD, Guerrero-Esteo M, Bernabeu C, Vary CP. Endoglin, a TGF-beta receptor-associated protein, is expressed by smooth muscle cells in human atherosclerotic plaques. Atherosclerosis. 2000;153 (2):323-335.

11. Venkatesha S, Toporsian M, Lam C, et al. Soluble endoglin contributes to the pathogenesis of preeclampsia. Nat Med. 2006;12 (6):642-649. doi:10.1038/nm1429

12. Levine RJ, Lam C, Qian C, et al.; CPEP Study Group. Soluble endoglin and other circulating antiangiogenic factors in preeclampsia. N Engl J Med. 2006;355(10):992-1005.

13. Buda V, Andor M, Ledeti A, et al. Comparative solid-state stability of Perindopril active substance vs. pharmaceutical formulation. Int J Mol Sci. 2017;18(1):E164. doi:10.3390/ijms18010164

14. Buda V, Andor M, Petrescu L, et al. Perindopril induces TSP-1 expression in hypertensive patients with endothelial dysfunction in chronic treatment. Int $J$ Mol Sci. 2017;18(2):E348. doi:10.3390/ ijms 18020348

15. Buda V, Andor M, Cristescu C, et al. The influence of perindopril on PTX3 plasma levels in hypertensive patients with endothelial dysfunction. Farmacia. 2016;64(3):382-389.

16. Kapur NK, Morine KJ, Letarte M. Endoglin: a critical mediator of cardiovascular health. Vasc Health Risk Manag. 2013;9:195-206. doi:10.2147/VHRM.S29144

17. Ikemoto T, Hojo $\mathrm{Y}$, Kondo $\mathrm{H}$, et al. Plasma endoglin as a marker to predict cardiovascular events in patients with chronic coronary artery diseases. Heart Vessels. 2012;27(4):344-351. doi:10.1007/s00380011-0163-z

18. Li CG, Bethell H, Wilson PB, Bhatnagar D, Walker MG, Kumar S. The significance of CD105, TGFbeta and CD105/TGFbeta complexes in coronary artery disease. Atherosclerosis. 2000;152 (1):249-256.

19. Casula M, Montecucco F, Bonaventura A, et al. Update on the role of pentraxin 3 in atherosclerosis and cardiovascular diseases. Vascul Pharmacol. 2017;99:1-12. doi:10.1016/j.vph.2017.10.003

20. Torsney E, Charlton R, Parums D, Collis M, Arthur HM. Inducible expression of human endoglin during inflammation and wound healing in vivo. Inflamm Res. 2002;51(9):464-470.

21. Kapur NK, Heffernan KS, Yunis AA, et al. Usefulness of soluble endoglin as a noninvasive measure of left ventricular filling pressure in heart failure. Am $J$ Cardiol. 2010;106(12):1770-1776. doi:10.1016/j.amjcard.2010.08.018

22. Kapur NK, Wilson S, Yunis AA, et al. Reduced endoglin activity limits cardiac fibrosis and improves survival in heart failure. Circulation. 2012;125(22):2728-2738. doi:10.1161/CIRCULATION AHA.111.080002

23. Prieto $M$, Rodríguez-Peña $A$, Arévalo $M$, et al. Effect of the long-term treatment with trandolapril on endoglin expression in rats with experimental renal fibrosis induced by renal mass reduction. Kidney Blood Press Res. 2005;28(1):32-40. doi:10. $1159 / 000081439$

24. Rathouska J, Vecerova L, Strasky Z, et al. Endoglin as a possible marker of atorvastatin treatment benefit in atherosclerosis. Pharmacol Res. 2011;64(1):53-59. doi:10.1016/j.phrs.2011.0 3.008 


\section{Publish your work in this journal}

Drug Design, Development and Therapy is an international, peerreviewed open-access journal that spans the spectrum of drug design and development through to clinical applications. Clinical outcomes, patient safety, and programs for the development and effective, safe, and sustained use of medicines are a feature of the journal, which has also

been accepted for indexing on PubMed Central. The manuscript management system is completely online and includes a very quick and fair peer-review system, which is all easy to use. Visit http://www. dovepress.com/testimonials.php to read real quotes from published authors.

Submit your manuscript here: https://www.dovepress.com/drug-design-development-and-therapy-journal 